\title{
Direct Numerical Simulation of Turbulent Heat Transfer Modulation in Micro-Dispersed Channel Flow *
}

\author{
Francesco Zonta, Cristian Marchioli and Alfredo Soldati \\ Centro Interdipartimentale di Fluidodinamica e Idraulica and Dipartimento di Energetica e Macchine, \\ Università degli Studi di Udine, 33100, Udine, Italy
}

\begin{abstract}
The object of this paper is to study the influence of dispersed micrometer size particles on turbulent heat transfer mechanisms in wall-bounded flows. The strategic target of the current research is to set up a methodology to size and design new-concept heat transfer fluids with properties given by those of the base fluid modulated by the presence of dynamically-interacting, suitablychosen, discrete micro- and nano- particles.

We run Direct Numerical Simulation (DNS) for hydrodynamically fully-developed, thermallydeveloping turbulent channel flow at shear Reynolds number $R e_{\tau}=150$ and Prandtl number $\operatorname{Pr}=3$, and we tracked two large swarms of particles, characterized by different inertia and thermal inertia. Preliminary results on velocity and temperature statistics for both phases show that, with respect to single-phase flow, heat transfer fluxes at the walls increase by roughly $2 \%$ when the flow is laden with the smaller particles, which exhibit a rather persistent stability against non-homogeneous distribution and near-wall concentration. An opposite trend (slight heat transfer flux decrease) is observed when the larger particles are dispersed into the flow. These results are consistent with previous experimental findings and are discussed in the frame of the current research activities in the field. Future developments are also outlined.
\end{abstract}

PACS numbers:

\section{INTRODUCTION}

The problem of developing efficient heat transfer techniques for technological applications has become more and more important over the last decades due to the increasing demand of cooling in high heat flux equipments and to the unprecedented pace of component miniaturization $[1,2,3]$. Consider, for instance, teraflop computers and other electronic equipments like optical fibers, high energy density lasers and high power x-rays. These devices are required to operate with high precision and, at the same time, with minimum size. Such requirements impose a challenge in terms of both device design and thermal management, not only in the case of micro-scale applications but also for large-scale applications such as transport vehicle engines, fuel cells and controlled bio-reactors [3]. Common air-based cooling systems have proven inadequate in high heat flux applications and more efficient techniques for heat transferability are thus required. In particular, a quest for a fluid with high heat transfer capacity, characterized by the possibility of tunable thermal properties and also associated to low management/safety problems has started. One possibility is to use nanofluids, namely dilute liquid suspensions of nanoparticulate solids including particles, nanofibers and nanotubes, which are supposed to change

\footnotetext{
* It is our great pleasure to take part in this Festschrift Issue dedicated to Professor Franz Ziegler on the occasion of his 70th birthday. This study was crafted with friendship and respect to honour his activity and his scientific achievements. We wish Franz many more productive, enjoyable and happy years and a solid and long collaboration as Editors of Acta Mechanica.
}

the heat transfer capabilities of the base fluid up to the target, desired amount. Nanofluids were first brought into attention approximately a decade ago, when their enhanced thermal behavior was observed with respect to conventional single-phase fluids such as water, engine oil, and ethylene glycol [3]. Specifically, due to the high thermal conductivity of metals in solid form, fluids containing suspended metal particles display significantly enhanced thermal conductivity and specific heat capacity $[4,5]$ compared to the conventional heat transfer liquids. For example, the thermal conductivity of gold at room temperature is more than 500 times larger than that of water and more than 2000 times larger than that of engine oil.

The idea of increasing the effective thermal conductivity of fluids with suspensions of solid particles is not so recent since the first theoretical formulation of nanofluids as a new concept of heat transfer fluids was put forth by Maxwell [6] more than one century ago, then followed by several other experimental and theoretical studies, such as those of Hamilton and Crosser [7] and Wasp et al. [8]. As of today, however, a clearcut understanding of the modifications of the heat transfer mechanisms occurring in nanofluids is still to be produced. This is indicated by the failure of classical models of suspensions and slurries in predicting nanofluids behavior [3,9] accompanied by the lack of alternative modelling strategies. Such lack may be possibly due to the number of investigations on the physical mechanisms which govern the heat transfer processes, still relatively low if compared to the corrispondingly large number of theoretical and experimental studies devoted to the problem of enhancing heat transferability with nanofluids (see [3,9] for a review). A possible way to improve the understanding of the physical transfer mechanisms is to use accu- 
rate and reliable numerical tools such as Direct Numerical Simulation (DNS) and Lagrangian Particle Tracking (LPT), which may complement complex and costly experiments. DNS-based Eulerian-Lagrangian studies have been widely used for investigating mass, momentum and heat transfer mechanisms in turbulent boundary layers. In particular, previous DNS studies on turbulent particle dispersion in wall-bounded flows (see [10-12] among others) have proven their capability of predicting particleturbulence interactions: these studies made it possible to perform phenomenological and quantitative analyses on the dispersion processes $[13,14]$, highlighting clustering and segregation phenomena. In the process of transferring this research methodology to nanofluids, one question requiring clarification for clearcut expectations is to what extent the same approach is proper to solve the different new physics challenges imposed by the different phenomena and range of parameters.

In this work, we propose to start approaching nanofluids from a simplified simulation setting where microparticles, rather than nanoparticles, are dispersed into the flow. This simplification allows to single out complications arising when the particles are very small and compare with the molecular scales of the fluid: inter-particle forces, wall effects, van der Waals forces, Brownian diffusion, etc. DNS has been used already to investigate on turbulent heat transfer in wall-bounded flows [15-21] and available studies provide systematic analyses of the Reynolds and Prandtl number effects on the heat transfer process. However, all these studies consider either singlephase turbulent flows $[16,18-21]$ or turbulent flows laden with coarse particles $[15,17]$. A comprehensive analysis accounting for mass, momentum and heat transfer mechanisms all together and tailored for the specific case of micro- or nano-dispersed fluids is currently unavailable as far as our knowledge goes. This is due to the non-trivial modeling issues, which of course reflect upon the complex interactions between the two phases. To elaborate, studying heat transfer modifications requires modeling particles as active heat transfer agents which interact both with the temperature field and the velocity field. Necessary energy and momentum coupling terms must be incorporated in the governing equations of both phases. This paper represents an effort toward a systematic phenomenological study of turbulent heat transfer mechanisms in micro- and/or nano-dispersed fluids. Since the modeling of these fluids represents a largely unexplored field of research, this study involves substantial challenges due to the rich complexity of the involved physics. The main focus of the present paper is to examine the modifications produced by solid inertial particles on the temperature fields of both fluid and particles. First, the numerical methodology that we use to investigate on the problem will be described; then preliminary statistical results will be shown and discussed in the limit of hydrodynamically fully-developed, thermally developing turbulent channel flow laden with particles large enough to neglect Brownian diffusion (which becomes important only for particle diameters smaller than $1 \mu \mathrm{m}$ ) but small enough to ensure stability against the inertia-dominated non-homogeneous distribution [12] and consequent near-wall accumulation $[10,13]$.

\section{METHODOLOGY}

\section{A. Flow field equations}

With reference to the schematics of Fig. 1, particles are introduced in a turbulent channel flow with heat transfer. Assuming that the fluid is incompressible and Newtonian, the governing balance equations for the fluid (in dimensionless form) read as:

$$
\begin{gathered}
\frac{\partial u_{i}}{\partial x_{i}}=0 \\
\frac{\partial u_{i}}{\partial t}=-u_{j} \frac{\partial u_{i}}{\partial x_{j}}+\frac{1}{R e^{*}} \frac{\partial^{2} u_{i}}{\partial x_{j}^{2}}-\frac{\partial p}{\partial x_{i}}+\delta_{1, i}+f_{2 w}, \\
\frac{\partial T}{\partial t}+u_{j} \frac{\partial T}{\partial x_{j}}=\frac{1}{R e^{*} \operatorname{Pr}} \frac{\partial^{2} T}{\partial x_{j}^{2}}+q_{2 w},
\end{gathered}
$$

where $u_{i}$ is the $i^{t h}$ component of the velocity vector, $p$ is the fluctuating kinematic pressure, $\delta_{1, i}$ is the mean pressure gradient that drives the flow, $T$ is the temperature, $R e^{*}$ is the shear (or friction) Reynolds number and $P r$ is the Prandtl number. The shear Reynolds number is defined as $R e^{*}=u^{*} h / \nu$, based on the shear velocity, $u^{*}$, on the half channel height, $h$, and on the fluid kinematic viscosity, $\nu$. The shear velocity is defined as $u^{*}=\left(\tau_{w} / \rho\right)^{1 / 2}$, where $\tau_{w}$ is the mean shear stress at the wall and $\rho$ is the fluid density. The Prandtl number is defined as $\operatorname{Pr}=\mu c_{p} / k$ where $\mu, c_{p}$ and $k$ are the dynamic viscosity, the specific heat and the thermal conductivity of the fluid, respectively. All variables considered in this study are reported in dimensionless form, represented by the superscript + , which has been dropped from Eqns. (1) to (3) for ease of reading, and expressed in wall units. Wall units are obtained by taking $u^{*}, \nu$ and the shear (or friction) temperature $T^{*}$ as the reference quantities employed for normalization. The shear temperature is defined as $T^{*}=q_{w} / \rho c_{p} u^{*}$ where $q_{w}=k \cdot \nabla T_{w}$ is the mean heat flux at the wall and $\nabla T_{w}$ is the wall-normal component of the temperature gradient at the wall. In Eqns. (2) and (3) the momentum-coupling term $f_{2 w}$ and the energy-coupling term $q_{2 w}$ are defined in terms of momentum and energy flux per unit mass, namely $f_{2 w}=F_{2 w} / m_{p}$ and $q_{2 w}=Q_{2 w} /\left(c_{p} \cdot m_{p}\right)$ where $m_{p}$ is the particle mass. These terms are introduced to model, as point sources, the influence of the particles on the fluid velocity and temperature fields (two-way coupling approach). More details on these terms are given in Sec. II C. The reference geometry consists of two infinite flat parallel walls; the origin of the coordinate system is located at the center of the channel and the 


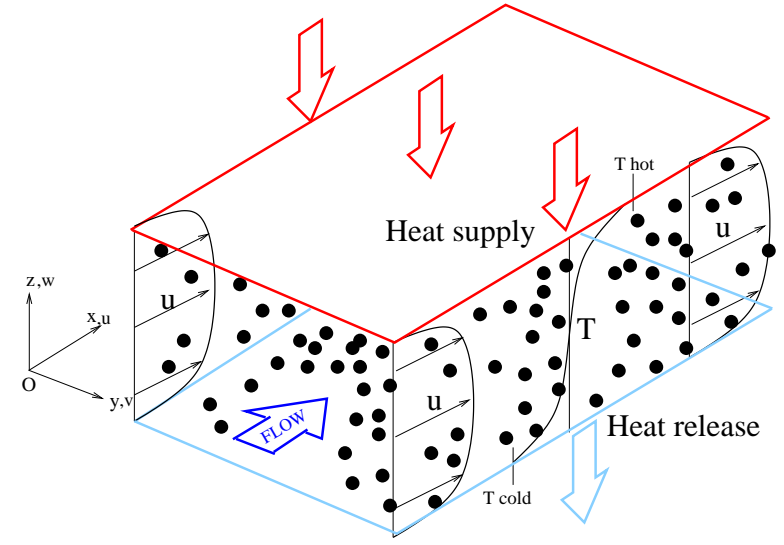

Figure 1: Sketch of the computational domain.

$x-, y-$ and $z-$ axes point in the streamwise, spanwise and wall-normal directions respectively (see Fig. 1). The calculations are performed on a computational domain of size $4 \pi h \times 2 \pi h \times 2 h$ in $x, y$ and $z$, respectively. Periodic boundary conditions are imposed on both velocity and temperature in the homogeneous $x$ and $y$ directions; at the wall, no-slip condition is enforced for the momentum equation whereas constant temperature condition is adopted for the energy equation. Specifically, the temperature on the boundaries is held constant at the uniform values $\left\langle T_{w}^{h}\right\rangle=80^{\circ} C$ for the hot wall (source of heat) and $\left\langle T_{w}^{c}\right\rangle=20^{\circ} C$ for the cold wall (sink of heat), where $<>$ denotes averaged values. The condition on temperature has been chosen because our aim is to simulate the problem of thermally-developing forced convection in a channel where supply of heat from a source and release of heat to a sink are considered as constant temperature processes.

\section{B. Particle equations}

Large samples of heavy particles with diameter $d_{p}=$ $4 \mu \mathrm{m}$ and $8 \mu \mathrm{m}$ and with density $\rho_{p}=19.3 \cdot 10^{3} \mathrm{~kg} \mathrm{~m}^{-3}$ (gold in water) are injected into the flow at concentration high enough to have significant two-way coupling effects in both the momentum and energy equations but negligible particle-particle interactions. The particle dynamics is described by a set of ordinary differential equations for position, velocity and temperature. For particles heavier than the fluid $\left(\rho_{p} / \rho \sim 20\right.$, as in the present case), Armenio and Fiorotto [22] showed that the most significant force is Stokes drag. Other forces acting on the particles, such as hydrostatic force, Magnus effect, Basset history force and added mass force are not taken into account since they are assumed to be negligible (orders of magnitude smaller) because of the specific set of physical parameters of our simulations $[22,23]$. We also neglected the Brownian force, which is proportional to $1 / d_{p}^{5}$ and becomes important for particle diameters less than $1 \mu \mathrm{m}$ [24]. With the above assumptions, a simplified version of the Basset-Boussinesq-Oseen equation for particle momentum balance [25] is obtained. In vector form:

$$
\begin{gathered}
\frac{d \mathbf{x}_{\mathbf{p}}}{d t}=\mathbf{u}_{\mathbf{p}}, \\
\frac{d \mathbf{u}_{\mathbf{p}}}{d t}=-\frac{3}{4} \frac{C_{D}}{d_{p}}\left(\frac{\rho}{\rho_{p}}\right)\left|\mathbf{u}_{\mathbf{p}}-\mathbf{u}\right|\left(\mathbf{u}_{\mathbf{p}}-\mathbf{u}\right),
\end{gathered}
$$

where $\mathbf{x}_{\mathbf{p}}$ is the particle position, $\mathbf{u}_{\mathbf{p}}$ is the particle velocity, $\mathbf{u}$ is the fluid velocity at particle position and $C_{D}$ is the drag coefficient. The formulation of the drag coefficient $C_{D}$ follows the non-linear approximation reported in Schiller and Naumann [26]:

$$
C_{D}=\frac{24}{R e_{p}}\left(1+0.15 R e_{p}^{0.687}\right),
$$

where $R e_{p}=d_{p}\left|\mathbf{u}_{\mathbf{p}}-\mathbf{u}\right| / \nu$ is the particle Reynolds number based on the relative particle-to-fluid velocity. The correction for $C_{D}$ is necessary when $R e_{p}$ does not remain small $\left(R e_{p}>1\right)$.

In this study, the particles exchange both momentum and heat with the carrier fluid. The equation describing the temperature evolution of the dispersed phase can be derived from the energy balance on a particle, written under the assumption of convective heat transfer occurring through the particle surface (the contribution of radiation is thus neglected). The inter-phase heat transfer rate of a spherical particle in motion relative to the surrounding fluid can be written as:

$$
\dot{Q}_{c}=N u \cdot \pi d_{p} \cdot k_{f} \cdot\left(T_{s}-T\right),
$$

where $N u$ is the Nusselt number, $k_{f}$ is the thermal conductivity of the fluid, $T$ is the temperature of the fluid and $T_{s}$ is the temperature at the particle surface. The Nusselt number is given by the well-known RanzMarshall correlation [27]:

$$
N u=2+0.6 \cdot \operatorname{Re}_{p^{\frac{1}{2}}} \cdot \operatorname{Pr}^{\frac{1}{3}}
$$

and accounts for changes of the heat transfer rate due to the relative motion between the particle and the surrounding fluid. Rearranging Eqn. (7), one can write:

$$
\frac{d T_{p}}{d t}=\frac{N u}{2} \cdot \frac{\left(T_{f}-T_{p}\right)}{\tau_{T}},
$$

where $T_{f}$ is the temperature of the fluid at particle position, $T_{p}$ is the temperature of the particle and $\tau_{T}=$ $\left(c_{p} \rho_{p} d_{p}{ }^{2}\right) /\left(12 k_{f}\right)$ is the particle thermal response time. In this study, we considered uniform temperature inside the particle, namely $T_{p}=T_{s}$ : this assumption is justified for particles with Biot number smaller than 0.1 [25]. The particle Biot number is defines as:

$$
B i=\frac{h_{p} d_{p}}{2 \lambda},
$$


where $h_{p}$ and $k_{p}$ are the convective heat transfer coefficient and the thermal conductivity of the particle. In the present simulations, the particle Biot number is $\mathcal{O}\left(10^{-6}\right)$.

\section{Modeling of two-way coupling}

The source terms $f_{2 w}$ and $q_{2 w}$ in Eqns. (2) and (3) arise because of the momentum transfer due to the drag force on the particle and because of the convective heat transfer to/from the particle, respectively. Calculation of these coupling terms is done applying the action-reaction principle to a generic volume of fluid $\Omega$ containing a particle. Focusing on the $f_{2 w}$ term (the extension for the term $q_{2 w}$ is straightforward), we have:

$$
\int_{\Omega} \mathbf{f}_{2 w}(\mathbf{x}) d \Omega=-\mathbf{f}_{f l u},
$$

where $\mathbf{f}_{f l u}$ is the force exerted by the fluid on the particle. The term $\mathbf{f}_{2 w}$, which represents the feedback of the dispersed phase on the fluid, can be obtained by adding the contributions of each particle:

$$
\mathbf{f}_{2 w}=\sum_{p=1}^{n_{p}}\left(\mathbf{f}_{2 w}^{p}\right),
$$

where $n_{p}$ is the total number of particles. The evaluation of each contribution $\mathbf{f}_{2 w}^{p}$ is obtained using the point source approximation $[28,29]$ :

$$
\mathbf{f}_{2 w}^{p}=-\mathbf{f}_{f l u} \delta\left(\mathbf{x}-\mathbf{x}_{p}\right),
$$

where $\delta\left(\mathbf{x}-\mathbf{x}_{p}\right)$ is the Dirac delta function.

\section{DNS methodology}

In this study a DNS of fully-developed channel flow with heat transfer is performed. The governing equations for the fluid, Eqns. (1)-(3), are discretized using a pseudo-spectral method based on transforming the field variables into wavenumber space, using Fourier representations for the periodic (homogeneous) directions and a Chebyshev representation for the wall-normal (nonhomogeneous) direction. As commonly done in pseudospectral methods, the convective non-linear terms are first computed in the physical space and then transformed in the wavenumber space using a de-aliasing procedure based on the $2 / 3$-rule; derivatives are evaluated directly in the wavenumber space to maintain spectral accuracy. Time advancement of the equations is performed using an explicit two-stage Euler/AdamsBashforth scheme for convective terms and an implicit Crank-Nicolson method for the viscous terms. The time step used is $d t^{+}=0.045$ in wall units. More details on the numerical scheme can be found in [30].

DNS calculations were performed at $R e^{*}=150$, corresponding to a bulk (average) Reynolds number $R e_{b}=$ $u_{b} h / \nu=1900$, where $u_{b}$ is the bulk velocity. At this Reynolds number, two different values of the Prandtl number were considered (see Table I). First, a simulation at $\operatorname{Pr}=0.71$ (run $R 1$ in Table I) was performed to validate the flow solver against numerical data available in the literature [31] for the situation of turbulent flow of air $\left(\rho=1.3 \mathrm{~kg} \mathrm{~m}{ }^{-3}, \nu=15.7 \cdot 10^{-6} \mathrm{~m}^{2} / \mathrm{s}\right)$ at ambient temperature in a channel of half height $h=0.02 \mathrm{~m}$. In this simulation, we have $u^{*}=0.11775 \mathrm{~m} \mathrm{~s}^{-1}$ and $u_{b}=1.49 \mathrm{~m} \mathrm{~s}^{-1}$. Second, simulations at $\operatorname{Pr}=3$ (corresponding to runs $R 2$ to $R 4$ in Table I) has been performed to study heat transfer modifications in a particle-laden turbulent flow of water $\left(\rho=10^{3} \mathrm{~kg} \mathrm{~m}^{-3}, \nu=10^{-6} \mathrm{~m}^{2} / \mathrm{s}\right)$ at the temperature $\langle T\rangle=50^{\circ} \mathrm{C}$ in a channel of half height $h=500 \mu \mathrm{m}$. The temperature $\langle T\rangle$ corresponds to the mean temperature between $\left\langle T_{w}^{h}\right\rangle$ and $\left.T<_{w}^{c}\right\rangle$. In this case, $u^{*}=0.3 \mathrm{~m} \mathrm{~s}^{-1}$ and $u_{b}=3.8 \mathrm{~m} \mathrm{~s}^{-1}$.

The computational domain has dimensions $1885 \times$ $942 \times 300$ in wall units and was discretized using an Eulerian grid made of $128 \times 128 \times 129$ nodes (corresponding to $128 \times 128$ Fourier modes and to 129 Chebyshev coefficients in the wavenumber space). The grid spacing is uniform in the homogeneous directions and corresponds to spatial resolutions equal to $\Delta x^{+}=14.72$ and $\Delta y^{+}=7.35$; the nodes along the wall-normal direction are clustered near the wall corresponding to spatial resolutions from $\Delta z^{+}=0.0452$ at the wall to $\Delta z^{+}=3.68$ at the centerline. The wall-normal grid spacing is always smaller than the smallest local flow scale and, thus, it fulfills the requirements imposed by the point-particle approach (see Sec. IIE).

\section{E. Lagrangian particle tracking}

The complete set of equations which describes the time evolution of particle position, velocity and temperature in the turbulent flow field is given by Eqns. (4), (5) and (9). To solve for these equations, we have coupled the DNS flow solver to a Lagrangian tracking routine. The routine uses $6^{\text {th }}$-order Lagrangian polynomials to interpolate the fluid velocity components and the fluid temperature at particle position. The performance of the interpolation scheme is comparable to that of spectral direct summation and to that of an hybrid scheme which exploits $6^{\text {th }}$-order Lagrangian polynomials in the streamwise and spanwise directions and Chebyshev summation

\begin{tabular}{cccccccccc}
\hline \hline RUN & $R e^{*}$ & $\begin{array}{c}u^{*} \\
{[\mathrm{~m} / \mathrm{s}]}\end{array}$ & $\begin{array}{c}\operatorname{Pr} \\
{[\mu \mathrm{m}]}\end{array}$ & $\begin{array}{c}d_{p} \\
{[\mu s]+}\end{array}$ & $\begin{array}{c}\tau_{p} \\
{[\mu s]}\end{array}$ & $\begin{array}{c}S t \\
\left(=\tau_{p}^{+}\right)\end{array}$ & $\begin{array}{c}\tau_{T} \\
{[\mu s]}\end{array}$ & $\begin{array}{c}S t_{T} \\
\left(=\tau_{T}^{+}\right)\end{array}$ \\
\hline$R 1$ & 150 & 0.11775 & 0.71 & - & - & - & - & - & - \\
\hline$R 2$ & 150 & 0.3 & 3 & - & - & - & - & - & - \\
\hline$R 3$ & 150 & 0.3 & 3 & 4 & 1.2 & 17.16 & 1.56 & 5.49 & 0.5 \\
\hline$R 4$ & 150 & 0.3 & 3 & 8 & 2.4 & 68.62 & 6.24 & 2.196 & 2.0 \\
\hline \hline
\end{tabular}

Table I: Summary of the simulation parameters. 
in the wall-normal direction. A $4^{\text {th }}$-order Runge-Kutta scheme is used for time advancement of the particle equations. The timestep size is equal to that used for the fluid $\left(\delta t^{+}=0.045\right)$. The total tracking time was $t^{+}=2000$ for the $4 \mu \mathrm{m}$ particles and $t^{+}=1500$ for the $8 \mu \mathrm{m}$ particles. We remark here that these simulation times are not sufficient to achieve a statistically steady state for the particle concentration, yet they are long enough to obtain converged velocity and temperature statistics and to highlight qualitatively the effect of the particles on the heat transfer rate. Particles are treated as pointwise, rigid spheres (point-particle approach) and are injected into the flow at average mass fraction, $\Phi_{m}$, high enough to have a two-way coupling between the particles and the fluid $\left(\Phi_{m} \sim 10^{-2}\right)[32,33]$. Possible effects due to interparticle collisions are neglected. At the beginning of the simulation, particles are distributed randomly over the computational domain and their initial velocity and temperature are set equal to those of the fluid at the particle initial position. Periodic boundary conditions are imposed on particles moving outside the computational domain in the homogeneous directions, whereas perfectlyelastic collisions at the smooth walls are assumed when the particle center is less than one particle radius away from the wall. No specific boundary condition is needed for the particle temperature equation (Eqn. 9) since the integration of this equation follows the integration of the particle momentum equation (Eqn. 5) and it requires only the knowledge of the initial condition. In the simulations presented here, large samples of 800,000 particles have been considered for each value of $R e^{*}$ and $\operatorname{Pr}$. We remark here that tracking of $\mathcal{O}\left(10^{6}\right)$ particles twoway coupled with the fluid requires a huge computational effort in terms of both computational cost of the simulation and disk storage availability, considering also the rather long tracking times achieved in the simulations. Each particle sample is characterized by different values of the particle response times. Table I summarizes the complete set of parameters relevant to the simulations of particle dispersion, including the particle Stokes numbers, $S t$ and $S t_{T}$. The particle Stokes number corresponds to the non-dimensional particle response time and is obtained using the viscous timescale $\tau_{f}=\nu / u_{\tau}^{2}$ as reference. In the present study, we have $S t=\tau_{p}^{+}=\tau_{p} / \tau_{f}$ and $S t_{T}=\tau_{T}^{+}=\tau_{T} / \tau_{f}$.

\section{RESULTS AND DISCUSSION}

\section{A. Unladen turbulent channel flow with heat transfer}

In this paragraph we examine the statistics relative to velocity and thermal variables for the base case of unladen fluid. We examine the results relative to the simulations $R 1$ and $R 2$, performed at the same Reynolds number $\left(R e^{*}=150\right)$ and at Prandtl numbers equal to 0.71 and $=3$, respectively. Velocity and temperature statistics will be examined and compared against literature reference cases [19,31].

\section{Velocity field}

The mean velocity profile will not be shown since it collapses onto the logarithmic law of the wall perfectly [13] and matches previous results obtained in Refs. [19,31]. In Fig. (2) the root mean square (rms) of the fluctuations of the velocity components, $\left\langle u_{i, r m s}^{+}\left(z^{+}\right)\right\rangle$, are plotted as a function of the wall normal coordinate in wall units, $z^{+}$, and compared against the results of Kasagi and Iida [31]. The agreement is generally good showing small differences which may be due to marginal statistical sampling of the time series. In the two simulations relative to the base fluid only, the velocity field depends only on the pressure gradient (namely on the shear Reynolds number, $R e^{*}$ ) and it is not influenced by the value of the Prandtl number - forced convection.

\section{Temperature field}

The behavior of the fluid temperature averaged over the homogeneous directions ( $x$ and $y$ ) is shown in Fig. (3) for the two values of the Prandtl number, $\operatorname{Pr}=0.71$ and $P r=3$ respectively. In Fig. (3a), the temperature is made dimensionless in outer units, indicated by the superscript -, as follows:

$$
<T^{-}>=\frac{<T>-T_{m}}{\Delta T_{m}},
$$

where $T_{m}=\left(T_{H}+T_{C}\right) / 2$ is the average centerline temperature and $\Delta T_{m}=\left(T_{H}-T_{C}\right) / 2$ is the temperature difference between the walls. The average temperature is shown as a function of the wall normal coordinate made

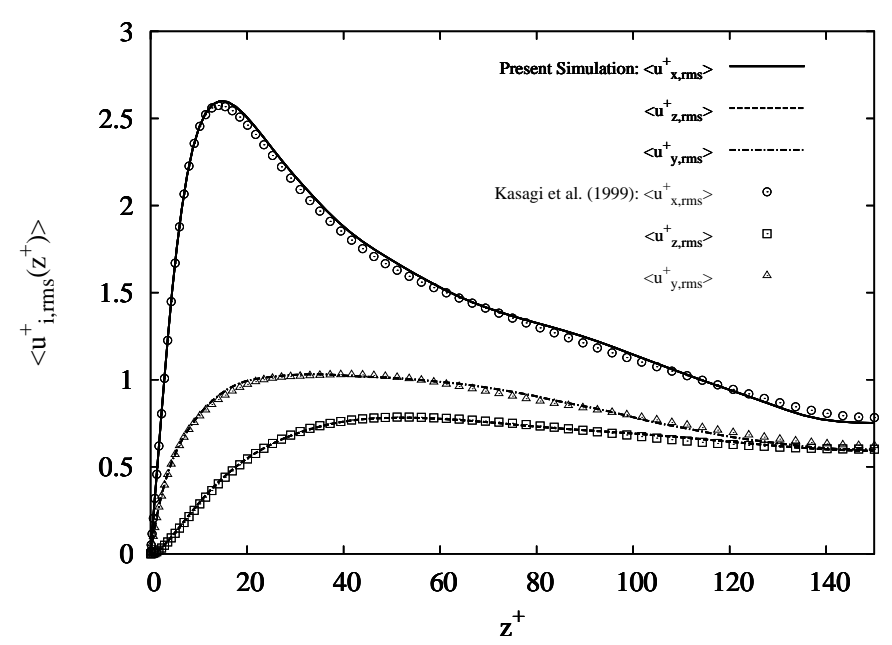

Figure 2: Rms of fluid velocity components, $\left\langle u_{i, r m s}^{+}\left(z^{+}\right)>\right.$, for single-phase (unladen) turbulent channel flow. 

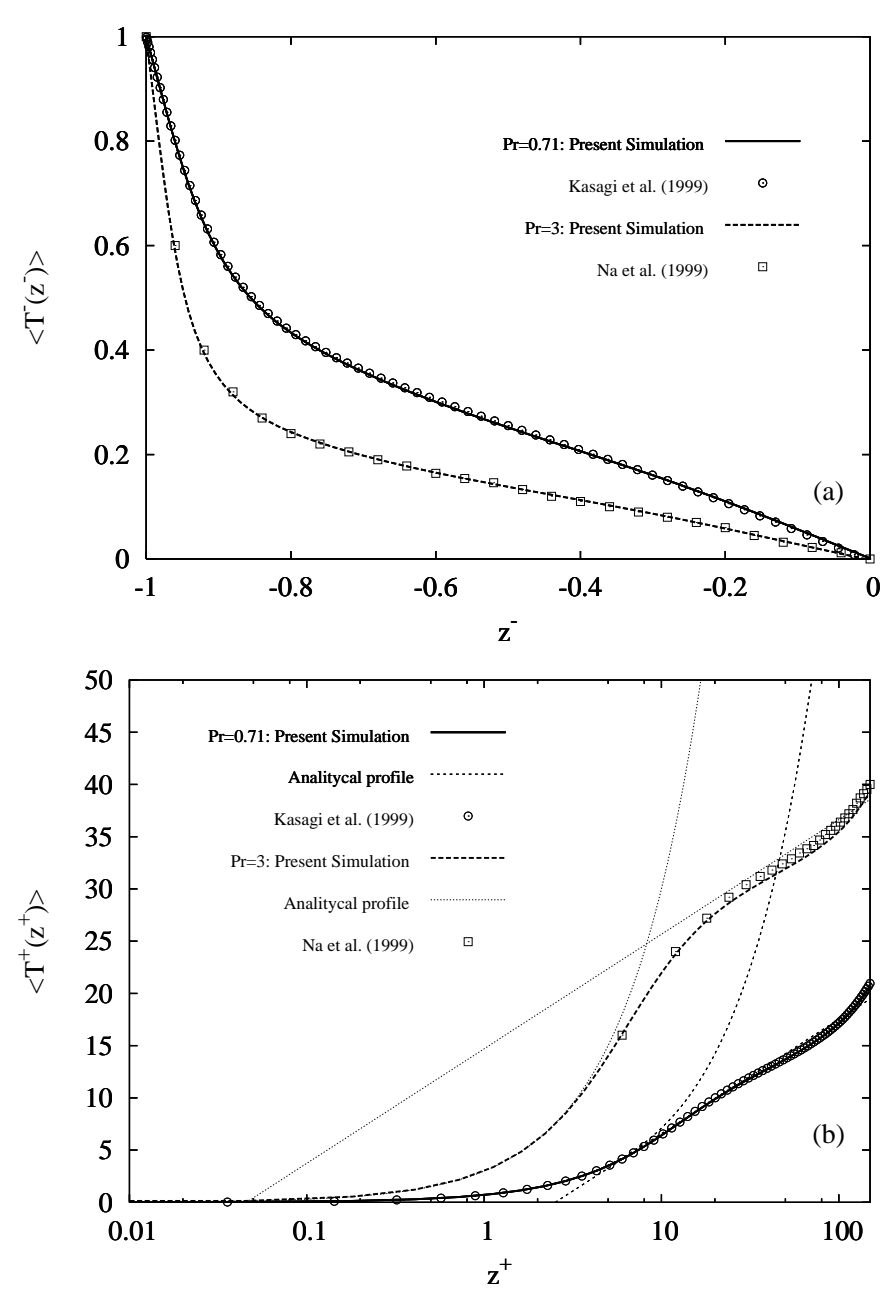

Figure 3: Mean fluid temperature at $\operatorname{Pr}=0.71$ and $\operatorname{Pr}=3$. Panels: (a) $<T^{-}\left(z^{-}\right)>$(linear-linear scale), $(\mathrm{b})<T^{+}\left(z^{+}\right)>$ (log-linear scale).

dimensionless by the half channel height, $h$. The results of the present work are compared and assessed against those computed by Kasagi and Iida [31] for $\operatorname{Pr}=0.71$ and against those computed by Na et al. [19] for $\operatorname{Pr}=3$. Results match perfectly showing that the simulation parameters - i.e. resolution, average time, etc. - are appropriate for the problem under investigation.

The same averaged temperature profiles are plotted in Fig. (3b) using a semi-logarithmic scale just to show possible minor differences among the current profiles and the benchmark profiles [19,31]. Temperature profiles are shown as a function of the wall normal distance, both being expressed in wall units. In this case, the temperature is defined as the difference between the local average temperature and the wall temperature (both relative to the average centerline temperature) normalized by the friction temperature, $T^{*}=q_{w} /\left(\rho_{f} c_{p} u^{*}\right)$ : $<T^{+}>=\left(<T>-T_{W}\right) / T^{*}$.

The computed data are also compared with standard analytical correlations used to estimate the temperature wall dependence. These correlations have the following form:

$$
<T^{+}>=\frac{<\bar{T}>-T_{W}}{T^{*}}= \begin{cases}P r \cdot z^{+} & \text {if } z^{+} \leq 11.6, \\ \frac{1}{k_{\theta}} \ln z^{+}+B_{\theta} & \text { if } z^{+}>11.6\end{cases}
$$

where $k_{\theta}=0.21$ and $B_{\theta}=-4.4$ and 14.7 for $\operatorname{Pr}=0.71$ and for $\operatorname{Pr}=3$, respectively.

As broadly known, the profiles show the existence of a (near-wall) diffusive sublayer, the thickness of which varies with the Prandtl number and is approximately equal to $\Delta \theta^{+}=6.5$ for $\operatorname{Pr}=0.71$ and $\Delta \theta^{+}=4.5$ for $\operatorname{Pr}=3$. In Figs. (3a) and (3b) we can further observe that the temperature gradient at the wall is strongly dependent on the value of the Prandtl number. This behavior is shown by Eqn. (15), from which it is clear that the temperature in the viscous sublayer depends linearly on the Prandtl number.

In problems which involve turbulent heat transfer, the Prandtl number is also important to establish the smallest spatial scale for the temperature field, $\eta_{\theta}$, which can be expressed $[34,35]$ as a function of the Kolmogorov scale, $\eta_{k}$, as follows:

$$
\eta_{\theta} \sim \eta_{k}\left(\frac{1}{\operatorname{Pr}}\right)^{3 / 4}
$$

for $\operatorname{Pr}<1$, and:

$$
\eta_{\theta} \sim \eta_{k}\left(\frac{1}{\operatorname{Pr}}\right)^{1 / 2}
$$

for $\operatorname{Pr}>1$. The previous relations confirm that for a given value of $\eta_{k}$ (we remind here that the average value of $\eta_{k}$ depends only on the Reynolds number, which is equal to 150 in the present simulations), the smallest temperature scale decreases for increasing Prandtl numbers.

The behavior of rms of the temperature field fluctuations, $\left\langle T_{r m s}^{+}\left(z^{+}\right)\right\rangle$, is shown in Fig. (4). Results obtained by Kasagi and lida [31] for $\operatorname{Pr}=0.71$ and by $\mathrm{Na}$ et al. [19] for $\operatorname{Pr}=3$ are also shown for benchmark and comparison. Results are presented in wall variables. Focusing on the $\operatorname{Pr}=0.71$ case, we observe that the temperature intensity reaches a maximum at the channel centerline, not mimicking the behavior of the fluctuations of the velocity field which all reach their peak in the wall proximity (see Fig. (2)). This was discussed by Lyons et al. [21], who attributed this different behavior to the temperature boundary conditions which force a non-zero temperature gradient at the center of the channel. In the $\operatorname{Pr}=3$ case, the temperature intensity reaches a maximum in the near-wall region. This observation is related to the Prandtl number effect on $\left\langle T_{r m s}^{+}(z)\right\rangle$, indicating that the range of wavenumbers in the thermal fluctuating field increases with $P r$, for which the spectral functions of the velocity fields are negligible. As can be also observed, the increase in the Prandtl number corresponds to a shift of the peak value of the temperature fluctuations toward the wall. Both in the current results and in 


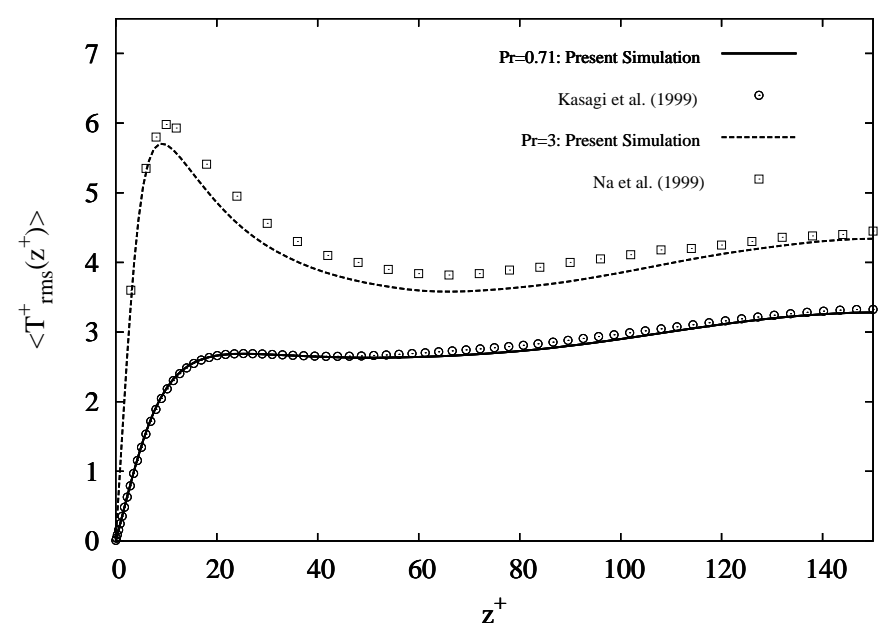

Figure 4: Rms of fluid temperature, $\left\langle T_{r m s}^{+}\left(z^{+}\right)\right\rangle$, at $\operatorname{Pr}=$ 0.71 and at $\operatorname{Pr}=3$.

those by Kasagi and Iida [31] for $P r=0.71$, the relative peak of the temperature fluctuations is located around $z^{+}=20$. The location of the peak for the higher Prandtl number $(\operatorname{Pr}=3)$ has moved to $z^{+}=9$, roughly. Small discrepancies due perhaps to the marginally statistical sample are observed between our results and those of $\mathrm{Na}$ et al. [19].

\section{B. Particle-laden turbulent channel flow with heat transfer and momentum/energy two way coupling}

The main purpose of this section is to analyze the modifications on the flow field due to the mutual interaction between fluid and particles. In particular, results obtained from two-way coupling simulations at $\mathrm{Pr}=3$ are compared against those obtained from the corresponding one-way coupling simulations, in which particle feedback on the flow is neglected: $f_{2 w}=0$ and $q_{2 w}=0$ in Eqns. (2) and (3). We remark here that, even though the problem of fluid-particle momentum coupling in twophase flows has been widely investigated $[11,28,33]$, much less effort has been devoted to the energy coupling problem. Currently, numerical studies on this problem are available for homogeneous shear flow [36] and for homogeneous isotropic turbulence [37]. To our knowledge, this is the first attempt to study (by means of DNS) both momentum and energy coupling between fluid and particles in wall-bounded flow.

\section{Velocity field modifications by particles}

The effect of particles on the mean fluid velocity, $<u_{x}^{+}\left(z^{+}\right)>$, is shown in Fig. (5). The solid line refers to the simulation without particles, while the dot-dashed line refers to the two-way coupling simulation with the $4 \mu \mathrm{m}$ particles. Profiles are averaged in space (over the

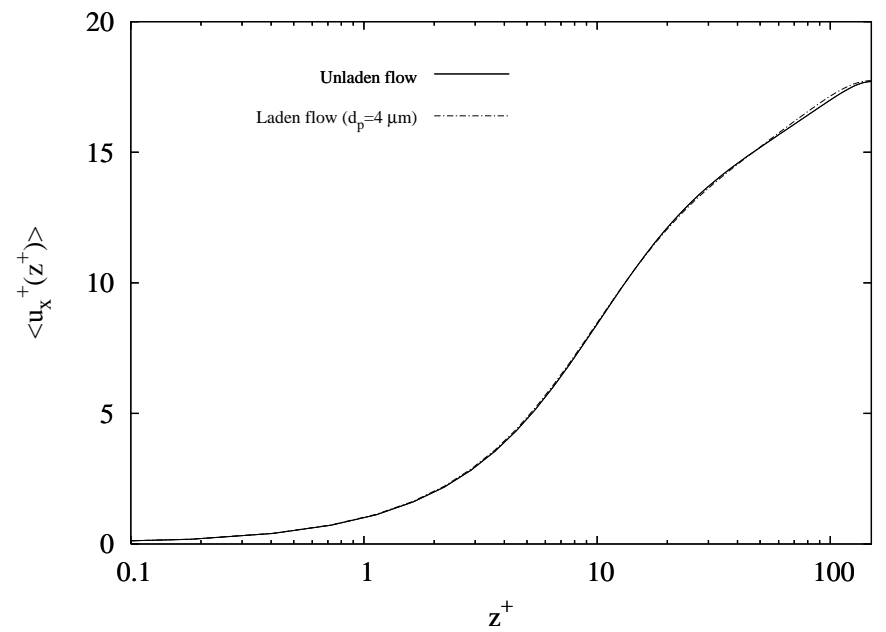

Figure 5: Mean streamwise fluid velocity, $\left\langle u_{x}^{+}\left(z^{+}\right)\right\rangle$, at $\operatorname{Pr}=3$ : comparison between unladen flow (no momentum/energy coupling) and flow laden with $d_{p}=4 \mu \mathrm{m}$ particles (with momentum/energy coupling).

streamwise and spanwise directions) and time (over a time span of 180 wall units). As expected, mean velocity profiles, normalized here by the unladen flow shear velocity $u^{*}$, deviate only slightly (if not negligibly, as in the viscous sublayer) from each other. A careful examination of Fig. (5) indicates that the effect of particles is to shift the velocity profiles slightly toward smaller values in the buffer region $\left(5<z^{+}<30\right)$ and toward higher values in the outer region $\left(z^{+}>30\right)$. Comparison of the mean velocity profiles for the $8 \mu \mathrm{m}$ particle case (not shown here for brevity) indicate no observable effect.

The behavior of the turbulence intensities, given by the rms of the fluid velocity fluctuations, $\left\langle u_{i, r m s}^{+}\left(z^{+}\right)\right\rangle$, shows larger differences as presented in Fig. (6). Again, the solid lines refer to the simulation without particles, whereas the dot-dashed and the dashed lines refer to the two-way coupling simulation with the $4 \mu \mathrm{m}$ particles and with the $8 \mu \mathrm{m}$ particles, respectively. It appears that particles do not affect much the intensities in the near-wall region but do substantially change them in the buffer region, particularly where the profiles develop a peak, and at the channel centerline. For each rms component, local changes of opposite sign depending on particle inertia are observed with respect to the reference unladen-flow values.

\section{Temperature field modifications by particles}

Fig. (7) shows the mean fluid temperature profiles in inner units, $\left\langle T^{-}\left(z^{-}\right)>\right.$, (Fig. 7a) and in wall units, $<T^{+}\left(z^{+}\right)>$(Fig. 7b). Lines are as in Fig. (5). Visual inspection of Fig. (7a) does not reveal significant differences in the profiles. However, computing the local value of the wall-normal temperature gradient, $\mathrm{d}\langle T(z)>/ \mathrm{d} z$, right at the wall, we obtain an increase of roughly $2 \%$ 

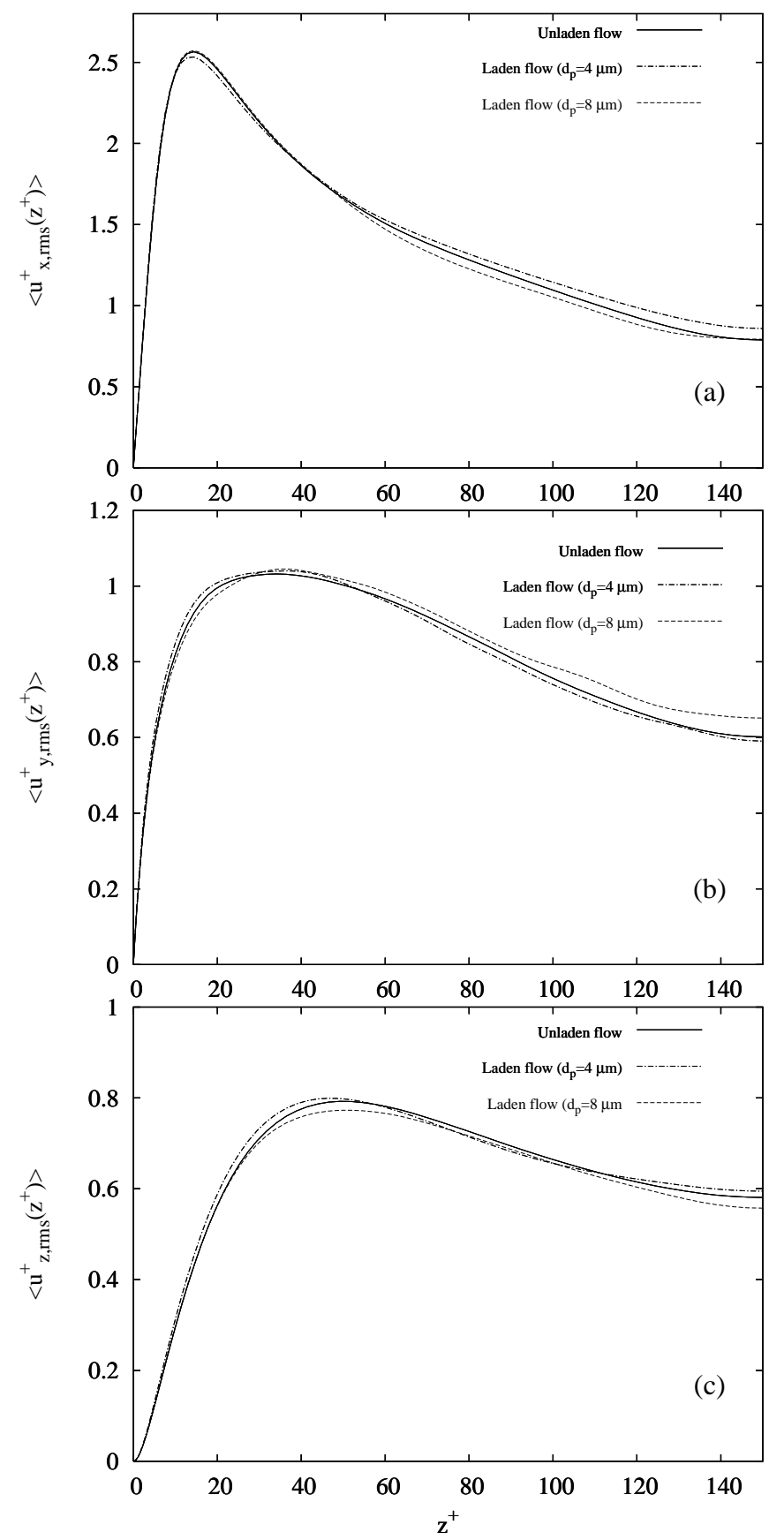

Figure 6: Rms of mean fluid velocity components, < $u_{i, r m s}^{+}\left(z^{+}\right)>$, at $\operatorname{Pr}=3$ : comparison between unladen flow (no momentum/energy coupling) and flow laden with $d_{p}=4 \mu \mathrm{m}$ particles and with $d_{p}=8 \mu \mathrm{m}$ particles (with momentum/energy coupling). Panels: (a) streamwise rms, $<u_{x, r m s}^{+}\left(z^{+}\right)>$; (b) spanwise rms, $<u_{y, r m s}^{+}\left(z^{+}\right)>$; (c) wallnormal rms, $<u_{i, r m s}^{+}\left(z^{+}\right)>$.

for the two-way coupling simulation with the $4 \mu \mathrm{m}$ particles and a decrease of $0.2 \%$ for the two-way coupling simulation with the $8 \mu \mathrm{m}$ particles. This finding is important because changes produced by the particles to the wall-normal temperature gradient directly reflect upon the heat flux at the wall, $q_{w}$, through the following
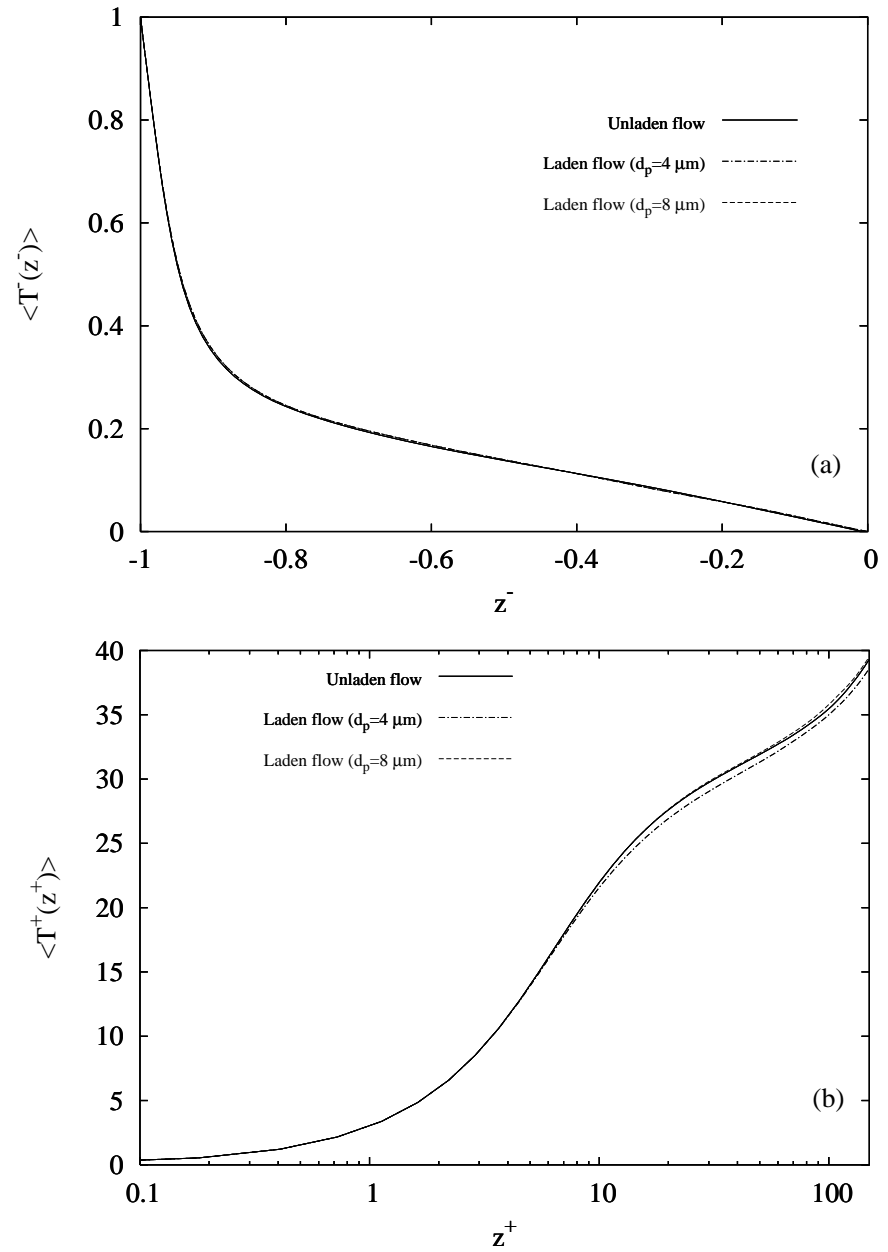

Figure 7: Mean fluid temperature at $\operatorname{Pr}=3$ : comparison between unladen flow (no momentum/energy coupling) and flow laden flow with $d_{p}=4 \mu \mathrm{m}$ particles and with $d_{p}=8$ $\mu m$ particles (with momentum/energy coupling). Panels: (a) $<T^{-}\left(z^{-}\right)>($lin-lin scale $),(\mathrm{b})<T^{+}\left(z^{+}\right)>(\log$-lin scale $)$.

expression:

$$
q_{w}=\frac{1}{\operatorname{Pr}} \frac{\mathrm{d}<T(z)>}{\mathrm{d} z} .
$$

In turn, a change in the value of $q_{w}$ will eventually correspond to a change in the value of the total turbulent heat flux $q_{z, t o t}$, defined as:

$$
q_{z, t o t}=q_{w}-<T^{\prime} w^{\prime}>,
$$

under fully developed conditions. Besides being perhaps the most significant result of the present paper from a quantitative viewpoint, the increase of removable heat flux at the wall for the smaller $4 \mu \mathrm{m}$ particles case and the decrease of removable heat flux for the larger $8 \mu \mathrm{m}$ particles case have also an effect on the friction temperature, $T^{*}$. According to its definition (see Sec. III A), the friction temperature computed in the two-way coupling simulations with the $4 \mu \mathrm{m}$ particles will increase with respect to the unladen flow case, whereas it will decrease 


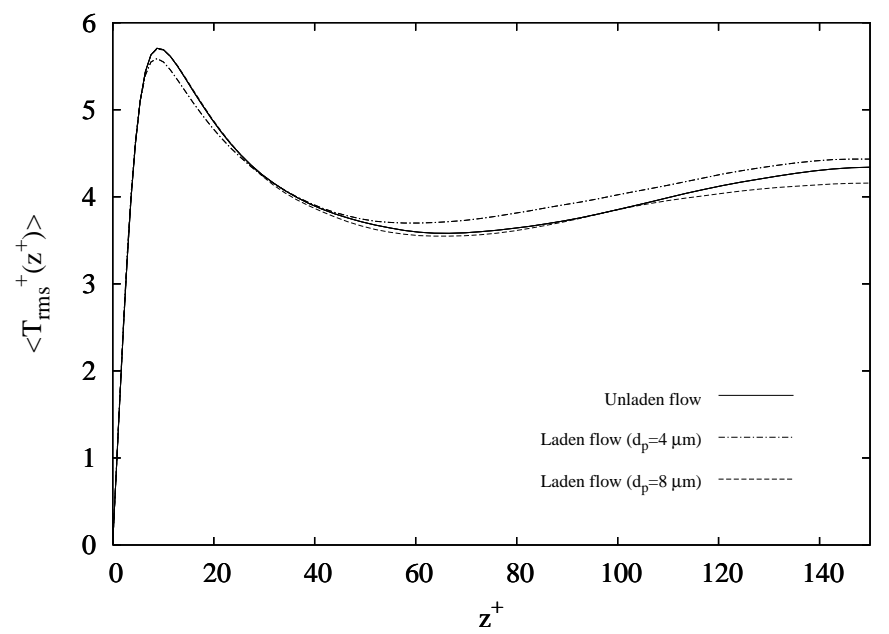

Figure 8: Rms of fluid temperature, $\left\langle T_{r m s}^{+}\left(z^{+}\right)\right\rangle$, at $\operatorname{Pr}=$ 3: comparison between unladen flow (no momentum/energy coupling) and flow laden with $d_{p}=4 \mu \mathrm{m}$ particles and with $d_{p}=8 \mu m$ particles (with momentum/energy coupling).

in the simulations with the $8 \mu \mathrm{m}$ particles. As a consequence, starting from the reference profile relative to the unladen flow case, the mean fluid temperature profile computed for the $4 \mu \mathrm{m}$ particle case will shift toward smaller values (particularly outside the viscous sublayer) whereas it will shift toward slightly higher values when the larger $8 \mu \mathrm{m}$ particles are considered, as apparent from Fig. (7b).

Finally, the rms of the fluid temperature fluctuations, $<T_{r m s}^{+}\left(z^{+}\right)>$, are shown in Fig. (8). The $4 \mu m$ particles produce a slight decrease in the peak value of $<T_{r m s}^{+}\left(z^{+}\right)>$and an increase outside the buffer region; smaller modifications (mostly limited to the core region of the flow) are produced by the larger $8 \mu \mathrm{m}$ particles.

\section{Influence of particle inertia and of particle thermal inertia}

The interactions between particles, turbulent momentum transport and turbulent heat transport are influenced by the particle response times. In this section we analyze the Eulerian statistics of the particles in comparison with those of the fluid. Specifically, particles will acquire and lose momentum and heat at a rate proportional to the inverse of their response times, $\tau_{p}$ and $\tau_{T}$ respectively.

Fig. (9) shows the mean streamwise velocity profile, $<u_{x, p}^{+}\left(z^{+}\right)>$, averaged in time and along the homogeneous directions, for both fluid and particles as a function of the wall-normal coordinate, $z^{+}$. Differences are readily visible, with a consistent evidence of the effect of particle inertia. Smaller particles $\left(S t=1.5, d_{p}=4 \mu \mathrm{m}\right)$ behave more like fluid tracers and their average velocity profile (circles) almost match that of the fluid (solid line). As the particle response time increases $\left(S t=6, d_{p}=8 \mu \mathrm{m}\right)$,

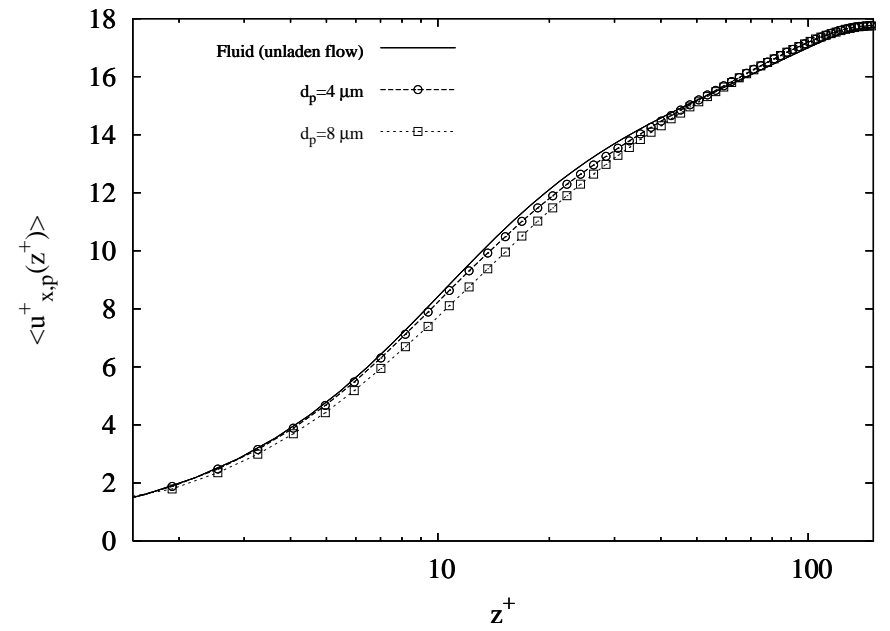

Figure 9: Mean particle streamwise velocity, $\left\langle u_{x, p}^{+}\left(z^{+}\right)\right\rangle$, at $\operatorname{Pr}=3$. Symbols: $(\bigcirc) d_{p}=4 \mu \mathrm{m}$ particles, $(\square) d_{p}=8 \mu \mathrm{m}$ particles. The mean fluid streamwise velocity profile relative to the unladen flow case (solid line) is also shown for sake of comparison.

the average particle velocity (squares) is seen to lag the average fluid velocity, in particular outside the viscous sublayer. Present results agree well with those reported by van Haarlem et al. [38] and by Portela et al. [39], who however used a one-way approach. This shows that, for the current size, density and overall concentration of the particles, modifications to the mean streamwise velocity profile due to momentum and energy coupling appear negligible. As already observed by van Haarlem et al. [38], inertial particles dispersed in a turbulent flow do not sample the flow field homogeneously and tend to avoid areas of high vorticity preferring areas characterized by lower-than-mean streamwise fluid velocity and by high strain rate. This gives the characteristic velocity lag in the region $5<z^{+}<50$. This effect is confirmed by data shown in Fig. (10a), where the root mean square of particles streamwise velocity (circles and squares) is compared to that of the fluid (solid line). Fluctuations of particle streamwise velocity are larger than those of the fluid, this difference becoming more evident as particle response time increases [39]. From a physical viewpoint, the difference in the streamwise values suggests that the gradients in the mean fluid velocity can produce significant fluctuations of the streamwise particle velocity. This effect seems more pronounced in the case of heavy particles, with larger Stokes number and a longer "memory". An opposite behavior is observed in the spanwise direction and in the wall-normal direction (Figs. (10b) and (10c), respectively), where the fluid velocity field has zero mean gradient. The turbulence intensity of the particles is very close to that of the fluid for particles with small inertia ( $S t=1.5$, circles) and lower than that of the fluid for particles with higher inertia ( $S t=6$, squares). This is mainly due to two mechanisms acting in tandem. The first mechanism is preferential concentration of particles 

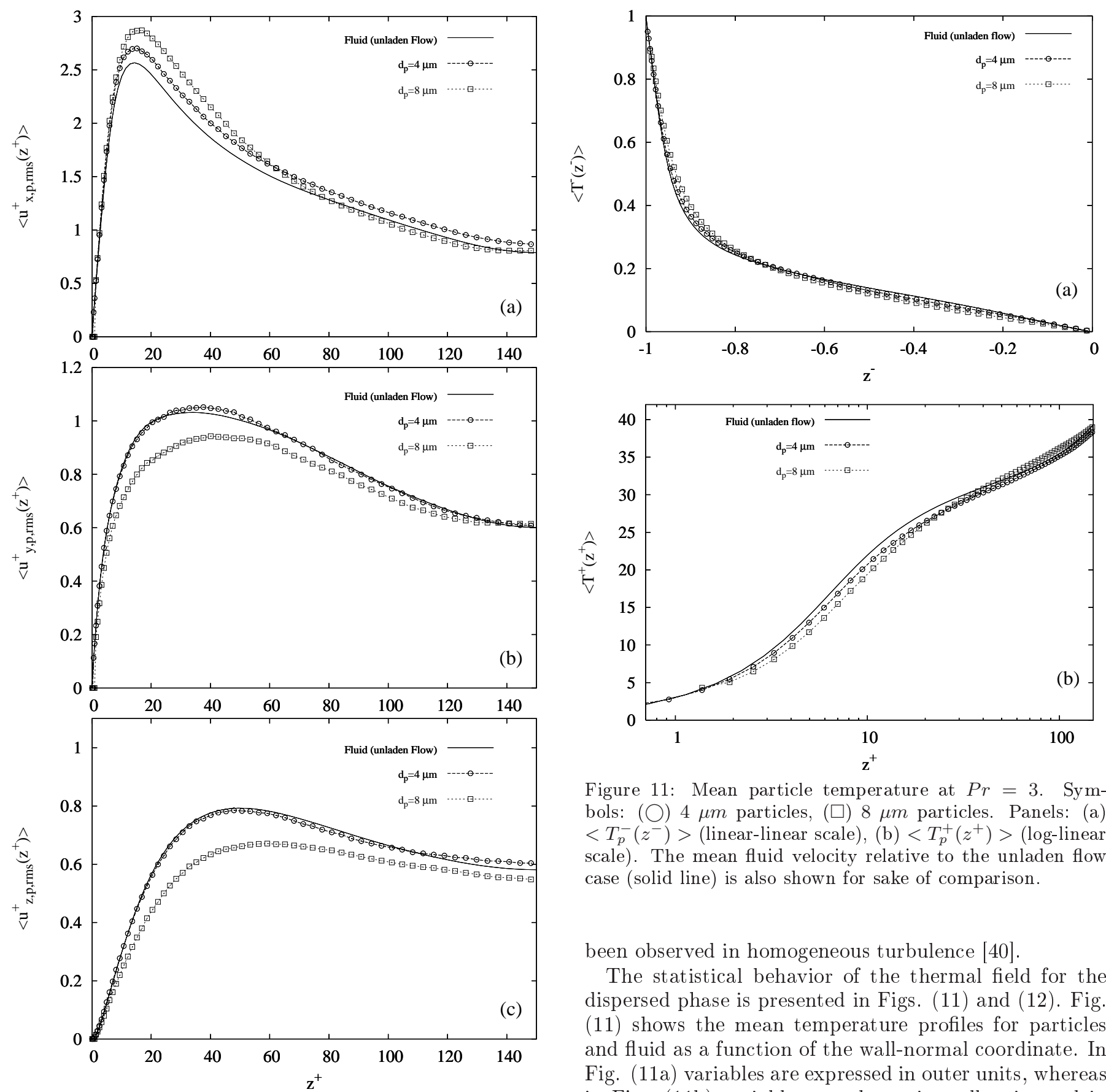

Figure 11: Mean particle temperature at $\operatorname{Pr}=3$. Symbols: (○) $4 \mu \mathrm{m}$ particles, ( $\square$ ) $8 \mu \mathrm{m}$ particles. Panels: (a) $<T_{p}^{-}\left(z^{-}\right)>$(linear-linear scale), $(\mathrm{b})<T_{p}^{+}\left(z^{+}\right)>$(log-linear scale). The mean fluid velocity relative to the unladen flow case (solid line) is also shown for sake of comparison.

been observed in homogeneous turbulence [40].

The statistical behavior of the thermal field for the dispersed phase is presented in Figs. (11) and (12). Fig. (11) shows the mean temperature profiles for particles and fluid as a function of the wall-normal coordinate. In Fig. (11a) variables are expressed in outer units, whereas in Fig. (11b) variables are shown in wall units and in semi-logarithmic scale. The solid line refers to the average unladen fluid temperature, while circles and squares refer respectively to the simulations with the $4 \mu \mathrm{m}$ particles and with the $8 \mu \mathrm{m}$ particles. Fig. (11a) indicates that, for both particle sets, the particle temperature in the near-wall region $\left(0<z^{+}<30\right)$ is higher than that of the fluid, while it reaches lower values in the outer region $\left(30<z^{+}<150\right)$. Particle temperature higher than that of the fluid is probably due to the deposition/resuspension mechanisms, which bring particles to the near-wall region (where they are characterized by high temperature value differences) and drive them toward the core region $[10,13]$. When a particle initially 


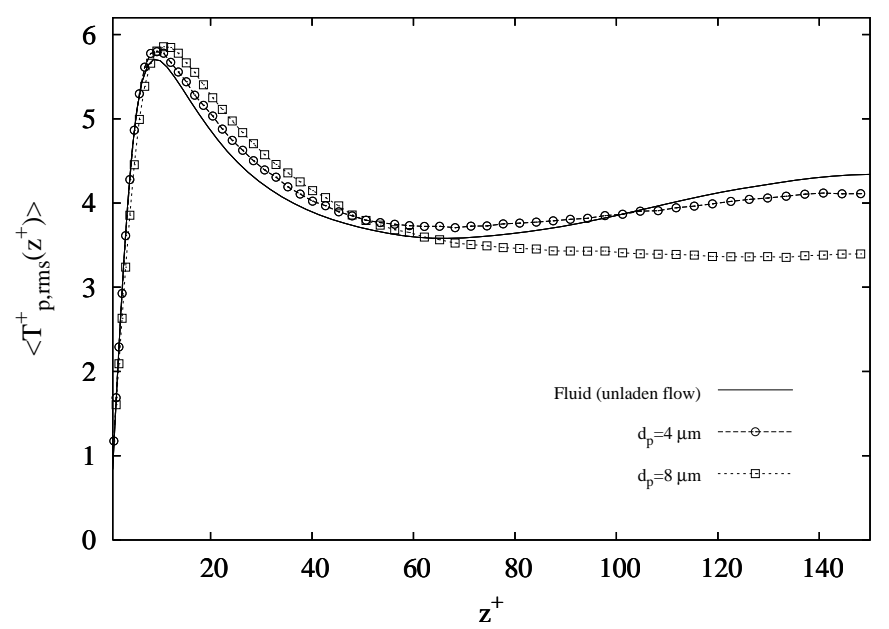

Figure 12: Rms of particle temperature, $\left\langle T_{p, r m s}^{+}\left(z^{+}\right)\right\rangle$, at $\operatorname{Pr}=3$. Symbols: $(\bigcirc) 4 \mu \mathrm{m}$ particles, $(\square) 8 \mu \mathrm{m}$ particles. The rms of fluid temperature relative to the unladen flow case (solid line) is also shown for sake of comparison.

close to the wall is entrained toward the core region, it will adapt its temperature at a rate depending on particle thermal inertia, thus maintaining a temperature higher than that of the fluid. In the outer region, the perspective is overturned, and particles are characterized by a temperature lower than that of the fluid. The effect of particle thermal inertia is also visible by observing the rms of particle temperature profiles, $\left\langle T_{p, r m s}^{+}\left(z^{+}\right)\right\rangle$, shown in Fig. (12). These profiles are qualitatively similar to those for the streamwise rms component (Fig. 10a), due to the role played by particle thermal inertia in transferring heat, similar to that played by particle inertia in transferring momentum. Although a qualitative similarity between the temperature and the streamwise velocity rms can be observed, quantitative differences are visible. In particular, the peak of the streamwise velocity rms exhibits values higher than the temperature rms. These quantitative differences are due to the fact that particle inertia is larger than particle thermal inertia and causes streamwise velocity fluctuations higher than thermal fluctuations.

\section{Instantaneous features of turbulent transfer of momentum, heat and particles}

A number of DNS-based works have been successful in clarifying the role of the instantaneous realizations of the Reynolds stresses in transferring momentum [41], heat [19] and particles [10]. We refer to these works and to the references therein for a more comprehensive description of the phenomena. It suffices here to show on a qualitative basis some relations among momentum, heat and particles transfer mechanisms.

To this aim, we complement the statistical analysis of the previous sections by showing the complex interac-
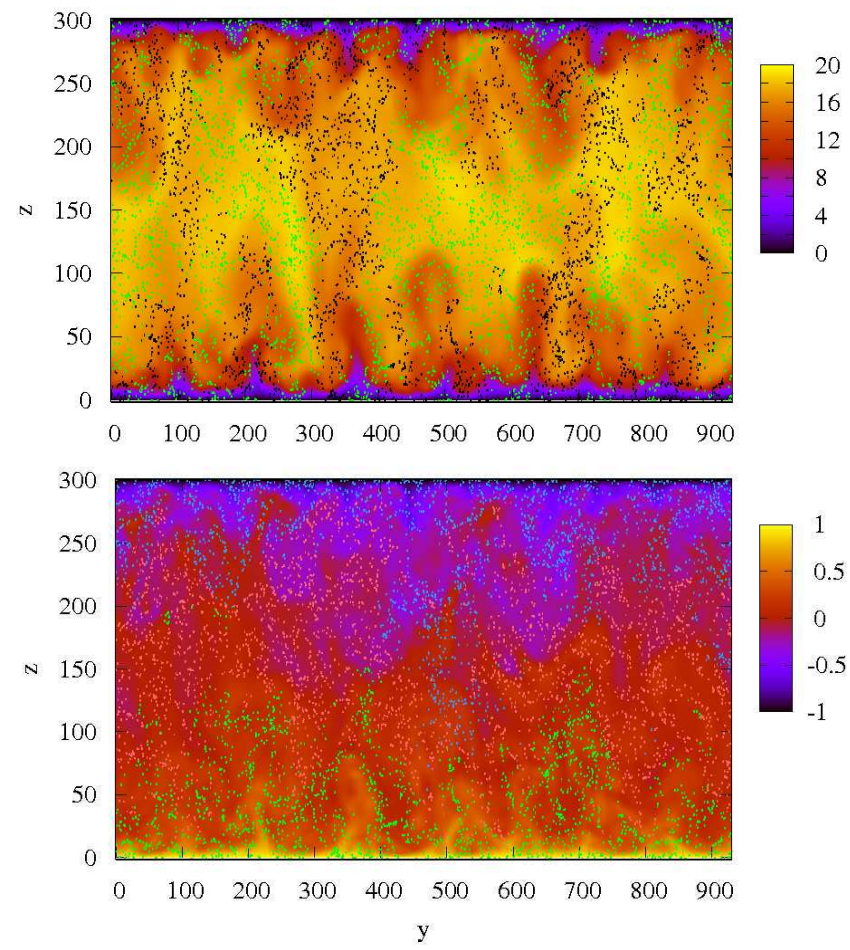

Figure 13: Front view of particle instantaneous distribution superimposed to the fluid velocity field (top) and to the fluid temperature field (bottom) at $\operatorname{Pr}=3$.

tions among velocity field, temperature field and particles distribution. In particular, we examine simulation $R 3\left(R e^{*}=150, P r=3\right.$ and $d_{p}=4 \mu m$ particles $)$. We consider a cross section of the flow field, perpendicular to the mean velocity, cut along a $x-y$ plane. Consider first Fig. (13a): color isocontours correspond to values of the streamwise fluid velocity whereas circles represent particles (drawn larger than the real size for visualization purposes) colored by their wall-normal velocity. In this Figure, green particles have wall-normal velocity directed toward the walls, while black particles have wall-normal velocity directed away from the walls. In this way, just by analyzing particle wall-normal velocity, it is possible to detect particle transfer fluxes toward the wall and particle transfer fluxes away from the wall. It is possible to observe that fluxes of particles are associated with fluxes of streamwise momentum (called sweeps if directed toward the wall and ejections if directed away from the wall, as discussed in several previous papers $[10,13])$.

To draw a link between the heat transfer mechanisms and the momentum transfer mechanisms we consider also Fig. (13b), where the particles are shown superimposed to the temperature field for the same section of Fig. (13a). In this case color isocontours are the values of the fluid temperature, whereas circles represent particles colored by their temperature: specifically, green particles have higher-than-mean temperature, blue particles have lower-than-mean temperature, and red particles have temperature close to the mean. The Reynolds 
transport analogy is clearly demonstrated by the behavior of the instantaneous temperature field: focusing on (a) the wall at $z^{+}=0$, characterized by higher temperature, we observe hot fluid plumes raising in correspondence with the raising of low momentum fluid within an ejection in Fig. (13a). This just demonstrates the effectiveness of the Reynolds stresses in transporting the fluid close to the wall towards the center of the channel and viceversa. Similar observations can be made for the cold wall at $z^{+}=300$. Considering now, again in Fig. (13b), the behavior of the particles in correlation with the temperature field, we observe that particles with higher temperature are ejected from the lower wall and are directed towards the upper wall. The opposite occurs to the colder particles. In this case, however, it is important to remind that particle temperature is not strictly correlated with fluid temperature. This behavior can be explained by considering that particle trajectories depend on fluid velocity and particle inertia, but not on fluid or particle temperature. Particles, driven by the fluid vortices, can thus reach regions characterized by fluid temperature quite different from particle temperature, causing an appreciable heat exchange between the two phases.

The efficiency of the overall heat exchange process is conditioned by the degree of non-homogeneity of particle distribution and by the rate of particle accumulation at the wall. This observation is qualitatively corroborated by Fig. (14), where the instantaneous cross-sectional distribution (left-hand side panels) and the wall-normal concentration, $C / C_{0}$ (right-hand side panels) of both particle sets are compared at the same time instant. Note that the $4 \mu \mathrm{m}$ particle distribution, shown in Fig. (14c), is the same of Fig. (13), the particle color code being that of Fig. (13b); particle concentration is computed as particle number density distribution per unit volume normalized by its initial value [10]. It is evident that the smaller $4 \mu \mathrm{m}$ particles, which produce an increase of heat transfer at the wall (as discussed in Sec. III B), exhibit a more persistent stability against non-homogeneous distribution and near-wall concentration with respect to the larger $8 \mu \mathrm{m}$ particles. The larger particles tend to form clusters in the core region and accumulate at the wall at higher rates acting as an additional thermal resistance both between the walls and the fluid and between the fluid and the particles: as a result of this behavior, the heat transfer is reduced.

\section{CONCLUSIONS AND FUTURE DEVELOPMENT}

Heat transfer enhancement is a fascinating subject with extremely interesting possibilities for application. One option to increase heat transfer is to devise a new concept of heat transfer media constituted by a base fluid in which suitably-chosen heat transfer agents, precisely micro and nano particles, are injected. In this way, the fluid can be a standard fluid characterized by simplicity of

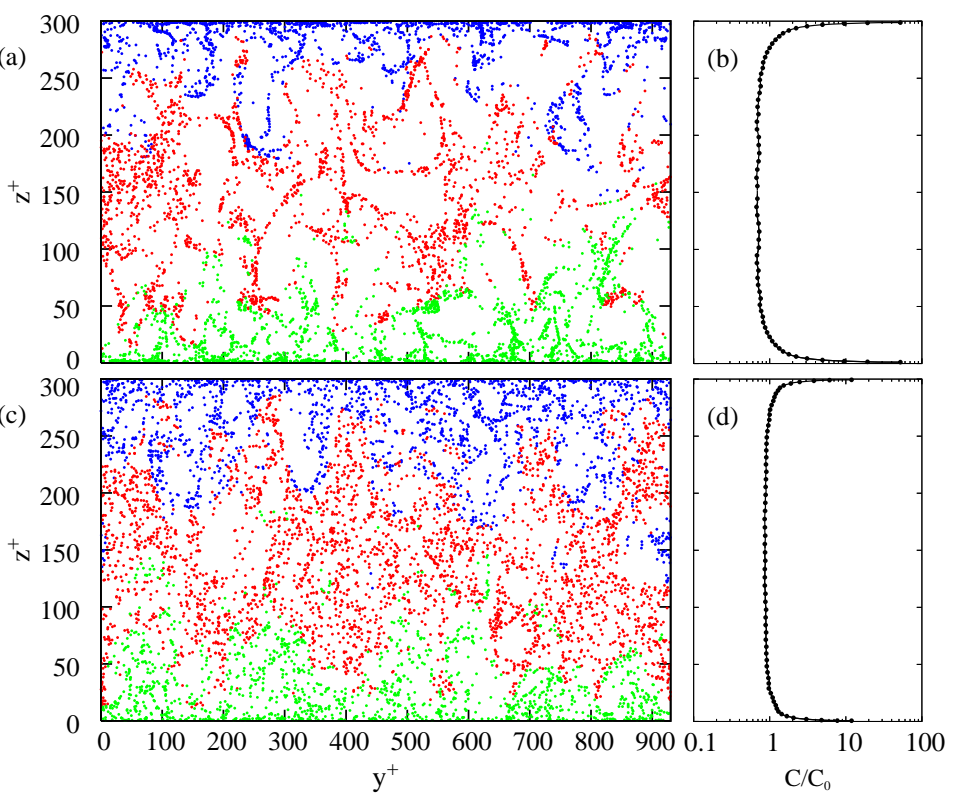

Figure 14: Front view of particle instantaneous distribution (left panels) and corresponding particle wall-normal concentration, $C / C_{0}$ (right panels) at $\operatorname{Pr}=3$. Panels: (a-b) $8 \mu \mathrm{m}$ particles, (c-d) $4 \mu \mathrm{m}$ particles.

use and well-known properties, like water, and the heat transfer agents can be heavy-metal, high-heat-capacity, dispersed particles (e.g. copper, gold or platinum).

Current literature trends show the potentials of such heat transfer media, called nanofluids; yet the complicacy and the cost of experimental methods make it hard to understand the intricacy of the mechanisms which govern the dynamics of the turbulent heat transfer among the fluid and the particles. Current open questions range from the optimal size of the particles, to the optimal concentration and practical solutions in real applications. Further complicating effects are represented by the particle inertia and the particle thermal inertia, which are additional parameters.

The present study represents a first effort made in the frame a broader project on the numerical simulation of heat transfer in nanofluids. Our strategic object is to investigate the heat transfer mechanisms in nanofluids and to devise a suitable numerical methodology to analyse their behavior. In this paper, the DNS of fluid and thermal fields is assessed against literature data (Kasagi and Iida [31] and $\mathrm{Na}$ et al. [19]) for one value of the shear Reynolds number, $R e^{*}=150$, and two values of the Prandtl number, $P r=0.71$ and $P r=3$. Further, preliminary results obtained from DNS of two-phase solid-liquid turbulent flow in a channel with heat transfer are presented. Hydrodynamically fully-developed, thermallydeveloping flow conditions have been considered to investigate on heat transfer modulation produced by the dispersion of micrometer sized particles. Two different sets of particles are considered, which are characterized by dimensionless inertia response times equal to $S t=1.56$ 
and $S t=6.24$ and by dimensionless thermal response times equal to $S t_{T}=0.5$ and $S t_{T}=2$, respectively. The instantaneous features of the velocity field, the temperature field and particles dispersion are discussed from a qualitative mechanistic viewpoint to analyse the role of particles as heat transfer enhancement agents.

Building on the results presented in this paper, an extensive numerical work combined with modeling efforts will be carried out to achieve a deeper understanding of the reason why nanofluids conduct heat so effectively. For instance, the increased surface interaction between the fluid and the solid particles at the nanoscale as possible explanation to the increased heat transferability will be investigated. Indeed, for a given volume of material there is a greater number of particles as their size decreases; perhaps there is more opportunity for the nanoparticles to conduct the heat. Furthermore, understanding of how the molecules of a base fluid keep nanoparticles suspended, since nanoparticles are still dramatically larger than individual molecules needs to be investigated. In this context, the effect of Brownian forces on the kinematics of the nanoparticles should be investigated. It would be also of interest to investigate the magnitude of the van der Waals forces between the particles and their effect on the nanofluids dynamics. These forces are usually small, but they become strong (and attractive) when the distance between particles becomes of the order of tenths of nanometers.

Acknowledgments Support from PRIN (under Grant 2006098584_004) and from HPC Europa Transnational Access Program (under Grants 466 and 708) are gratefully acknowledged.

\section{References}

1. Wang, X.-Q., Mujumdar, A.S.: Heat transfer characteristics of nanofluids: a review. Int. J. Therm. Sci. 46, 1-19 (2007).

2. Daungthongsuk, W., Wongwises, S.: A critical review of convective heat transfer of nanofluids. Renew. Sust. Energ. Rev. 11, 797-817 (2007).

3. Das, S.K., Choi, S.U.S., Patel, H.E.: Heat transfer in nanofluids - A review. Heat Transfer Eng. 27, 3-19 (2006).

4. Xuan, Y., Li, Q.: Heat transfer enhancement of nanofuids. Int. J. Heat Fluid Fl. 21, 58-64 (2000).

5. Maiga, S.E.B, Palm, S.J., Nguyen, C.T., Roy, G., Galanis, N.: Heat transfer enhancement by using nanofluids in forced convection flows. Int. J. Heat Fluid Fl. 26, 530-546 (2005).
6. Maxwell, J.C.: A treatise on electricity and magnetism, 2nd ed., vol. 1, Oxford: Clarendon Press, 1881, p. 435.

7. Hamilton, R.L., Crosser, O.K.: Thermal conductivity of heterogeneous two component systems. Ind. Eng. Chem. Fund. , vol. 1, 3, 187-191 (1962).

8. Wasp, E.J., Kenny, J.P., Gandhi, R.L.: Solidliquid flow slurry pipeline transportation. Series on book materials handling. Clausthal: Trans. Tech. Publications, 1977.

9. Trisaksri, V., Wongwises, S.: Critical review of heat transfer characteristics of nanofluids. Renew. Sust. Energ. Rev. 11, 512-523 (2007).

10. Marchioli, C., Soldati, A.: Mechanisms for particle transfer and segregation in turbulent boundary layer. J. Fluid Mech. 468, 283-315 (2002).

11. Pan, Y., Banerjee, S.: Numerical simulation of particle interaction with wall turbulence. Phys. Fluids 8, 2733-2755 (1996).

12. Eaton, J.K., Fessler, J.R.: Preferential concentration of particles by turbulence. Int. J. Multiphase Flow 20, 169-209 (1994).

13. Soldati, A.: Particles turbulence interactions in boundary layers. Z. Angew. Math. Mech. 85, 683-699 (2005).

14. Bec, J., Biferale, L., Boffetta, G., Celani, A., Cencini, M., Lanotte, A., Musacchio, S., Toschi, F.: Acceleration statistics of heavy particles in turbulence. J. Fluid Mech. 550, 349-358 (2005).

15. Chagras, V., Oesterlè, B., Boulet, P.: On heat transfer in gas-solid pipe flows: Effects of collision induced alteration of the flow dynamics. Int. J. Heat Mass Tran. 48, 1649$1661(2005)$.

16. Lakehal, D., Fulgosi, M., Yadigaroglu, G.: Direct numerical simulation of turbulent heat transfer across a mobile, sheared gas-liquid interface. J. Heat Transf. 125, 1129-1139 (2003).

17. Hetsroni, G., Mosyak, A., Pogrebnyak, E.: Effect of coarse particles in a particle laden turbulent boundary layer. Int. J. Multiphase Flow 28, 1873-1894 (2002).

18. Tiselj, I., Bergant, R., Mavko, B., Bajsić, Hetsroni, G.: DNS of turbulent heat transfer in channel flow with heat conduction in 
the solid wall. J. Heat Transf. 123, 849-857 (2001).

19. Na, Y., Papavassiliou, D.V., Hanratty, T.J.: Use of direct numerical simulation to study the effect of Prandtl number on temperature fields. Int. J. Heat Fluid Flow 20, 187-195 (1999).

20. Kawamura, H., Abe, H., Matsuo, Y.: DNS of turbulent heat transfer in channel flow with respect to Reynolds and Prandtl number effects. Int. J. Heat Fluid Fl. 20, 196-207 (1999).

21. Lyons, S.L., Hanratty, T.J., McLaughlin, J.B.: Direct numerical simulation of passive heat transfer in a turbulent channel flow. Int. J. Heat Mass Tran. 34, 1149-1161 (1991).

22. Armenio, V., Fiorotto, V.: The importance of the forces acting on particles in turbulent flows. Phys. Fluids 8, 2437 (2001).

23. Rizk, M.A., Elghobashi, S.E.: The motion of a spherical particle suspended in a turbulent flow near a plane wall. Phys. Fluids 28, 806817 (1985).

24. Soltani, M., Ahmadi, G.: Direct numerical simulation of particle entrainment in turbulent channel flow. Phys. Fluids A 7, 647-657 (1995).

25. Crowe, C., Sommerfeld, M., Tsuji, Y.: Multiphase Flows with Droplets and Particles. New York: CRC Press 1998, p. 469.

26. Schiller, V.L., Naumann, A.: Uber die grundlegenden Berechnungen bei der Schwerkraftaufbereitung. Z. Ver. Deut. Ing. 77, 318-320 (1935).

27. W. Ranz and W. Marshall: Evaporation from Drops. Chem. Eng. Progress, 48, 142-180 (1952).

28. Boivin, M., Simonin, O., Squires, K.D.: Direct numerical simulation of turbulence modulation by particles in isotropic turbulence. J. Fluid Mech. 375, 235-263 (1998).

29. Sundaram, S., Collins, L.R.: A numerical study of the modulation of isotropic turbulence by suspended particles. J. Fluid Mech. 379, 105-143 (1999).

30. Lam, K., Banerjee, S.: On the condition of streak formation in bounded flows. Phys. Fluids A 4, 306-320 (1992).
31. Kasagi, N. and Iida, O.: Progress in direct numerical simulation of turbulent heat transfer. Proceedings of the 5th ASME/JSME Joint Thermal Engineering Conference, In CD-ROM, March 15-19, San Diego, California (1999).

32. Uijttewaal, W.S., Oliemans, R.V.A: Particle dispersion and deposition in direct numerical and and large eddy simulations of vertical pipe flow. Phys. Fluids 8, 2590-2604 (1996).

33. Kiger, K.T., Pan, C.: Suspension and turbulence modification effects of solid particulates on a horizontal turbulent channel flow. J. Turbul. 3, 1-21 (2002).

34. Monin, A.S., Yaglom, A.M.: Statistical Fluid Mechanics:Mechanism of Turbulence, book 2, MIT Press, 1975, p. 873.

35. Batchelor, G.K.: Small-scale variation of convective quantities like temperature in turbulent fluid. J. Fluid Mech. A 5, 113-133 (1959).

36. Shotorban, B., Mashayek, F., Pandya, R.V.R.: Temperature statistics in particleladen turbulent homogeneous shear flow. Int. J. Multiphase Flow 29, 1333-1353 (2003).

37. Jaberi, F.A., Mashayek, F.: Temperature decay in two-phase turbulent flows. Int. J. Heat Mass Tran. 43, 993-1005 (2000).

38. van Haarlem, B., Boersma, B.J., Nieuwstadt, F.T.M : Direct numerical simulation of particle deposition onto a free-slip and no-slip surface. Phys. Fluids A. 10, 2608-2620 (1998).

39. Portela, L.M., Cota, P., Oliemans, R.V.A : Numerical study of the near wall behaviour of particles in turbulent pipe flows. Powder Technol. 125, 149-157 (2002).

40. Reeks, M.W.: The transport of discrete particles in inhomogeneous turbulence. J. Aerosol Sci. 310, 729-739 (1983).

41. Adrian, R.J., Meinhart, C.D., Tomkins, C.D.: Vortex organization in the outer region of the turbulent boundary layer. J. Fluid Mech. 422, 1-54 (2000). 